Syracuse University

SURFACE

1993

\title{
Multimedia Object Modelling and Storage Allocation Strategies for Heterogeneous Parallel Access Storage Devices in Real Time Multimedia Computing Systems
}

\author{
C.Y. Roger Chen \\ Syracuse University, Department of Electrical and Computer Engineering \\ Kingsley C. Nwosu \\ IBM POWER Parallel Systems \\ P. B. Berra \\ Syracuse University
}

Follow this and additional works at: https://surface.syr.edu/eecs

Part of the Computer Sciences Commons

\section{Recommended Citation}

Chen, C.Y. Roger; Nwosu, Kingsley C.; and Berra, P. B., "Multimedia Object Modelling and Storage Allocation Strategies for Heterogeneous Parallel Access Storage Devices in Real Time Multimedia Computing Systems" (1993). Electrical Engineering and Computer Science. 103.

https://surface.syr.edu/eecs/103

This Article is brought to you for free and open access by the College of Engineering and Computer Science at SURFACE. It has been accepted for inclusion in Electrical Engineering and Computer Science by an authorized administrator of SURFACE. For more information, please contact surface@syr.edu. 


\section{Multimedia Object Modelling and Storage Allocation Strategies for Heterogeneous Parallel Access Storage Devices in Real Time Multimedia Computing Systems}

\author{
C. Y. Roger Chen, \\ Dept. of Elect. and Comput. Eng., \\ Syracuse University, \\ Syracuse, NY 13244.
}

\author{
Kingsley C. Nwosu, \\ IBM POWER Parallel Systems, \\ MS/992, Neighborhood Rd., \\ Kingston, NY 12401.
}

\author{
P. Bruce Berra, \\ CASE Center, \\ Syracuse University, \\ Syracuse, NY, 13244.
}

\begin{abstract}
The improvements in disk speeds have not kept up with improvements in processor and memory speeds. Conventional storage techniques, in the face of multimedia data, are inefficient and/or inadequate. Here, an efficient multimedia object allocation strategy is presented. We describe a multimedia object model, the object and storage device characteristics, and the fragmentation strategy. A bipartite graph approach is used for mapping fragments to storage devices and a cost function is used to determine an efficient allocation of an object and to balance the loads on the devices.
\end{abstract}

Keywords: bipartite graphs, bipartite matching, efficient allocation, fragmentation, multimedia, storage allocation.

\section{Introduction}

The rapid advances in the technology of display devices, computers, networks, storage devices, and software engineering have pushed the emerging multimedia applications into becoming one of the most important and promising research areas. Multimedia information processing encompasses the integrated generation, representation, processing, storage, and dissemination of independent machine processable information expressed in multifarious time dependent and independent media. A unique feature of multimedia is the highly diversified media types and file sizes. In order to avoid dealing with the heterogeneity of multimedia data, multimedia applications are usually developed using an object-oriented approach, where each object represents a file of video, audio, image, graphics, text, etc; or a combination of them. Moreover, it is usually required to integrate or combine multiple objects of various media types into multiple-level complex objects. By using the object-oriented approach, multimedia data can be processed and manipulated by users in a universal way, regardless of the media types and sizes of objects. However, from a system's point of view, many problems arise in supporting such an object-oriented multimedia system. Among the problems, a most serious one is related to the storage. This is due to the fact that processor speed, memory speed, and memory size have grown exponentially over the past few years [1][2], while disk speeds have improved at a far slower rate. Consequently, the speed of the disk rather than the speed of the CPU's is the limiting factor in many applications. For real-time information retrieval and presentation, it is imperative that data, for a given medium, be retrievable at some given rate. The rates for some media are very high for current storage devices. The most conspicuous of these is in the area of digital video. For example, the video data object based on the NTSC standard requires that video data be retrievable at a rate of $45 \mathrm{Mbits} / \mathrm{sec}$. However, the peak speed of a magnetic disk drive is about $10 \mathrm{Mbits} / \mathrm{sec}$. and CD-ROMs operate at 1.2 Mbits/sec. To meet the bandwidth requirement of a full-motion video file, it is clear that the file has to be spilt into multiple sub-files, stored in different disks; when needed, an interleaving technique will be performed to combine the multiple data streams into a single data stream and then present it to the user.

Conventional allocation techniques (such as data stripping/de-clustering [3][4][5] and data contiguity/clustering [6][7][8]) are developed mainly for text and numeric files, which although can be different in sizes, are more or less on the same order. Unfortunately, when applied to multimedia applications, the conventional techniques are inadequate and inefficient. Several file system level approaches [9][10] have been proposed and utilized; however, they do not encompass the gamut of multimedia types and are mostly for continuous media types (digital audio and video) without addressing the storage allocation with empha- 
Figure 2: An example of object splitting.

\section{Multimedia object/storage charac- teristics and problem formulation}

The $j$ th DE in a composite object $O$ is denoted as $o_{j}$. Each DE is associated with a frequency distribution. This frequency distribution represents the relative probability that a given $\mathrm{DE}$ will be requested for retrieval. Each class-one DE, $o_{j}$, has an expected retrieval rate. This rate represents the minimum number of bytes of $o_{j}$ that should be retrieved per unit time in order to achieve its real-time requirements. Each class-three DE, $o_{k}$, is associated with a degree of parallelism which indicates the degree of concurrent access to the DE that may be helpful for computations for either multi-processors or vector processors.

Like the multimedia objects, the $k$ th storage device is denoted as $S_{k}$, its bandwidth as $B W\left(S_{k}\right)$, total amount of space already allocated as $S_{k}^{a}$, and the free space as $S_{k}^{f}$. The total number of storage devices is represented by $m$. Since we are dealing with a heterogeneous environment where the computing system comprises different types of storage devices with different characteristics, the differing characteristic that is of paramount importance to us is the bandwidth of a storage device. We group related devices together based on their bandwidths into $\wp^{1}, \wp^{2}, \ldots, \wp^{\mu}$, where $\mu$ is the number of different bandwidths in the system and the bandwidth of each storage device in $\wp^{i}$ is $B W\left(\wp^{i}\right)$. We denote the $k$ th $\mathrm{AU}$ of $o_{j}$ as $a_{j, k}$ and the total number of AUs in $o_{j}$ as $\alpha_{j}$. Consequently, the storage allocation problem is formulated as follows: (1) Given a composite multimedia object, how can one decompose the DEs to build the AUs such that the allocation of the AUs achieve the real time requirements? (2) Given a list of AUs produced from the fragmentation strategy, how does one define the

allocation process and AU allocatability? (3) Having determined the storage devices to which an $\mathrm{AU}$ is al- 
locatable, what criteria are necessary and sufficient in determining the most efficient storage device to store an AU? (4) Given an allocation strategy, how can one demonstrate that it fairly and sufficiently balances the loads among the storage devices?

\section{Problem and allocation analysis}

Having defined the problem, it is important that we discuss the vital decisions that must be made to address the facets of the allocation process. Each allocation process comprises a composite object with its associated DEs. The storable elements are the DEs. A composite object is dynamically created and stored in the system with respect to the current status of the storage devices. For an allocation process, all the DEs of a composite object are considered simultaneously. Since the most common operation during the allocation process is the determination of the mappings of AUs to storage devices, we denote the fact that AU a is mapped to $S_{k}$ as $a \models S_{k}$.

\subsection{Intra DE allocation}

The intra DE allocation stipulates the allocation policy that must exist when allocating the AUs of a DE. In the case of a class-one or class-three DE, it is imperative that each of its AUs be stored in a different storage device in order to achieve the expected retrieval rate or degree of parallelism. Therefore, if $o_{i}$ is a class-one or class-three DE, then the intra DE allocation states that

$$
\begin{gathered}
\forall_{k=1, \alpha_{i}} \text { if } a_{i, k}=S_{h} \text { then } \forall_{g=1, \alpha_{i} \quad \nexists a_{i, g} \text { such that }} \\
a_{i, g} \neq S_{h} \quad(k \neq g) \text { and }(1 \leq h \leq m) .
\end{gathered}
$$

\subsection{Intra complex object allocation}

As is prevalent in most complex object oriented systems, users or applications may need to access all the data associated with a complex object concurrently. In that case, therefore, it becomes necessary that all the DEs of a complex object be stored in such a way that all of its data can be retrieved concurrently. We must, therefore, allocate each AU of a complex object to a different storage device. Therefore, if $o_{1}, \ldots, o_{j}$ are the DEs of a complex object, then the intra complex object allocation states that

$$
\begin{gathered}
\forall_{x_{1}=1, j} \forall_{x_{2}=1, \alpha_{x_{1}}} \text { if } a_{x_{1}, x_{2}} \models S_{h} \text { then } \\
\forall_{x_{3}=1, j} \forall_{x_{4}=1, \alpha_{x_{3}}} \nexists a_{x_{3}, x_{4}} \text { such that } \\
a_{x_{3}, x_{4}} \models S_{h}, \quad\left(x_{3} \neq x_{1}, 1 \leq h \leq m\right) .
\end{gathered}
$$

\subsection{Inter composite object allocation}

Another access situation that we must consider is when the access crosses multiple composite objects. In an environment with a limitless number of storage devices, we can afford to store every $\mathrm{AU}$ in a different storage device. However, that situation is unrealistic. We, sometimes, expect the situation depicted in Figure 3 to occur where composite objects $O 1$ and $O 2$ share $O 1$ 's $o 2$. For allocation purposes, we logically think of shared DEs as, physically, belonging to each of the composite object. For example, using Figure 3 , if $O 1$ were allocated first, then none of $O 2$ 's AUs should be allocated in the same device with $O 1$ 's $o 2$.

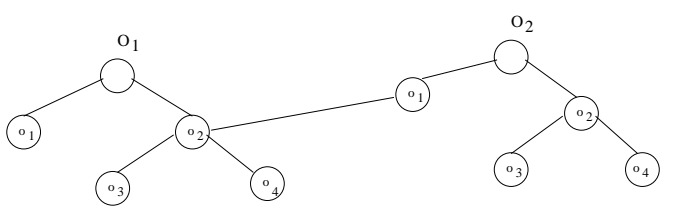

Figure 3: An example of object sharing.

\section{$5 \quad$ Fragmentation strategies}

For class-one DEs, we have to decompose their data into fragments that foster parallel reads to achieve their expected retrieval rates. In order to obtain these fragments, we have to determine the degree of decomposition of a given DE. We need to compute the number of storage devices that can be accessed in parallel to satisfy the retrievability requirement. A DE's Storage Set $(\chi)$ is the set of the number of storage devices needed to achieve its expected retrieval rate based on the amount of data that can be retrieved from each storage device per unit time (i.e., bandwidth) in parallel. If we have a homogeneous configuration of storage devices, then the computation of the number of storage devices needed is straightforward since all of the storage devices have the same bandwidth. In the case of heterogeneous storage devices, we have to consider different storage devices with different bandwidths. So we are forced to consider combinations of different storage devices with different bandwidths. Consequently, a DE can have multiple storage sets. An element of a storage set indicates the possible number of storage devices for one or more device clusters that is necessary to achieve the real time requirement of a DE. It is obvious that the number of sets in a storage set could become very large. As a result, some con- 
straints, as described below, are utilized to minimize the size of a storage set. To that end, therefore, the number of sets in a DE's storage set is reduced to at most $2^{\mu}-1$. Let $\chi_{k}=\left\{y^{1}, y^{2}, \ldots\right\}$ be the $k$ th storage set of $o_{j}$ where $y^{i}$ is the number of storage devices from $\wp^{i}$ needed to achieve the expected retrieval rate. For each combination of device clusters that form a storage set, each cluster must be represented by at least one storage device. The amount of data retrievable from the storage devices of a storage set must not be less than the expected retrieval rate or degree of parallelism, but should exceed that value with minimum value. The storage device clusters are arranged in order of decreasing bandwidths. The number of storage devices per device cluster in a storage set decreases with increasing bandwidth, when applicable. In the rest of the paper, when necessary, the expected retrieval rate of a class-one DE $o_{j}$ is represented as $\beta_{j}$. For example, given $\beta_{j}$ and $\chi_{k}=\left\{y^{1}, y^{2}, y^{3}\right\}$ then the following conditions must hold:

1. $B W\left(\wp^{1}\right)>B W\left(\wp^{2}\right)>B W\left(\wp^{3}\right)$,

2. $y^{1} \leq y^{2} \leq y^{3}$,

3. $\left[y^{1} B W\left(\wp^{1}\right)+y^{2} B W\left(\wp^{2}\right)+y^{3} B W\left(\wp^{3}\right)\right] \geq \beta_{j}$, and
(a) $\left[\left(y^{1}-1\right) B W\left(\wp^{1}\right)+y^{2} B W\left(\wp^{2}\right)+\right.$ $\left.y^{3} B W\left(\wp^{3}\right)\right]<\beta_{j}$,
(b) $\left[y^{1} B W\left(\wp^{1}\right)+\left(y^{2}-1\right) B W\left(\wp^{2}\right)+\right.$ $\left.y^{3} B W\left(\wp^{3}\right)\right]<\beta_{j}$,
(c) $\left[y^{1} B W\left(\wp^{1}\right)+y^{2} B W\left(\wp^{2}\right)+\left(y^{3}-\right.\right.$ 1) $\left.B W\left(\wp^{3}\right)\right]<\beta_{j}$.

If any of the conditions above is violated, then the corresponding storage set is invalid. The above conditions are equivalent to solving the integer linear programming problem:

$$
\begin{gathered}
y^{1} B W\left(\wp^{1}\right)+y^{2} B W\left(\wp^{2}\right)+y^{3} B W\left(\wp^{3}\right) \geq \beta_{j}, \\
y^{1} \leq y^{2}, y^{2} \leq y^{3}, y^{3}>0 .
\end{gathered}
$$

An $\chi_{k}$ with $\left|\chi_{k}\right|^{1}=g$ is acceptable if

(1) $\sum_{i=1}^{g} y^{i} \leq m$, and

(2) $\forall j=1, g y^{j} \leq\left|\wp^{j}\right|$.

For example, consider a class-one $\mathrm{DE}$ of size $120 \mathrm{~KB}$ and bandwidth requirement of $60 \mathrm{~KB} / \mathrm{s}$, given that $\wp^{1}=\left\{S_{1}, S_{2}, S_{3}\right\}, \wp^{2}=\left\{S_{4}, S_{5}\right\}, \wp^{3}=$ $\left\{S_{6}, S_{7}, S_{8}\right\}, B W\left(\wp^{1}\right)=30, B W\left(\wp^{2}\right)=20$, and $B W\left(\wp^{3}\right)=10$. The sets of the combinations of clusters of storage devices are $\left\{\wp_{1}\right\},\left\{\wp_{2}\right\},\left\{\wp_{3}\right\},\left\{\wp_{1}, \wp_{2}\right\},\left\{\wp_{1}, \wp_{3}\right\},\left\{\wp_{2}, \wp_{3}\right\}$, and $\left\{\wp_{1}, \wp_{2}, \wp_{3}\right\}$. The valid storage sets are $\chi_{1}=$

\footnotetext{
${ }^{1}|A| \rightarrow$ the number of elements in set $\mathrm{A}$
}

$\{2\}, \chi_{2}=\{3\}, \chi_{3}=\{6\}, \quad \chi_{4}=\{1,2\}, \chi_{5}=$ $\{1,3\}, \chi_{6}=\{2,2\}$, and $\chi_{7}=\{1,1,1\}$.

Obviously, $\chi_{2}$, and $\chi_{3}$ are not acceptable. Furthermore, without the constraints discussed above, for example, it is evident that given $\left\{\wp_{2}, \wp_{3}\right\}$, the storage sets $\{3,0\},\{1,4\},\{0,6\},\{2,3\}$, and $\{1,5\}$ can achieve the real time requirements. However, applying the constraints limits the option to $\{2,2\}$. If none of the storage sets of a DE is acceptable, then we can not allocate the DE. When that happens, a message may be sent to the user suggesting a higher degree of data compression on the class-one DEs or a lower degree of parallelism for class-three DEs. Since the size of each data retrieved per unit time from each storage device is its bandwidth, each AU stored in a storage device comprises a number of chunks of data whose size is equal to the bandwidth of the storage device. We call each of this chunk of data a Storage Element (SE). An $\mathrm{AU}$ then consists of one or more SEs arranged in such a way that guarantees parallel retrieval of contiguous data. For a given DE, we denote as $\gamma_{k}$ the number of storage devices in $\chi_{k}$. We call $\gamma_{k}$ the storage length of a storage set. Consequently, each $\chi_{k}$ comprises $\gamma_{k}$ AUs where each AU is denoted as $a_{k, l}, 1 \leq l \leq \gamma_{k}$. We represent the number of SEs in $a_{k, l}$ as $\delta_{k, l}$. The sum of all the sizes of all the SEs of all the AUs of a DE must be at least as large as the size of the DE. Furthermore, reducing any AU of any storage set of a DE by one SE must violate the preceding condition. We denote as $\aleph_{k, l}^{q}$ the fact that $\mathrm{SE} f_{q}$ belongs to AU $a_{k, l}$. Therefore, for $\chi_{k}$

$$
\text { if } \aleph_{k, l}^{q} \text { then } \forall_{\left(h \bmod \gamma_{k}=q \bmod \gamma_{k}\right)} \aleph_{k, l}^{h} \quad(h \neq q) \text {. }
$$

As is evident from building the AUs, the data represented by each $\mathrm{AU}$ do not constitute a contiguous data in a DE. The physical addresses of the SEs in an AU differ by some factors of the bandwidths of the storage devices. This is a consequence of data interleaving which is essential for achieving parallel I/O for a stream of data. Figure 4 shows the SEs and AUs of all the storage sets. The numbers beside the boxes represent the physical addresses of the SEs in a DE.

The above discussion on fragmentation strategy has been done in the context of class-one DEs. In the case of class-three DEs, the degree of parallelism also represents the expected number of AUs. Therefore, for a class-three DE, a storage set is valid if its storage length is equal to the DE's 


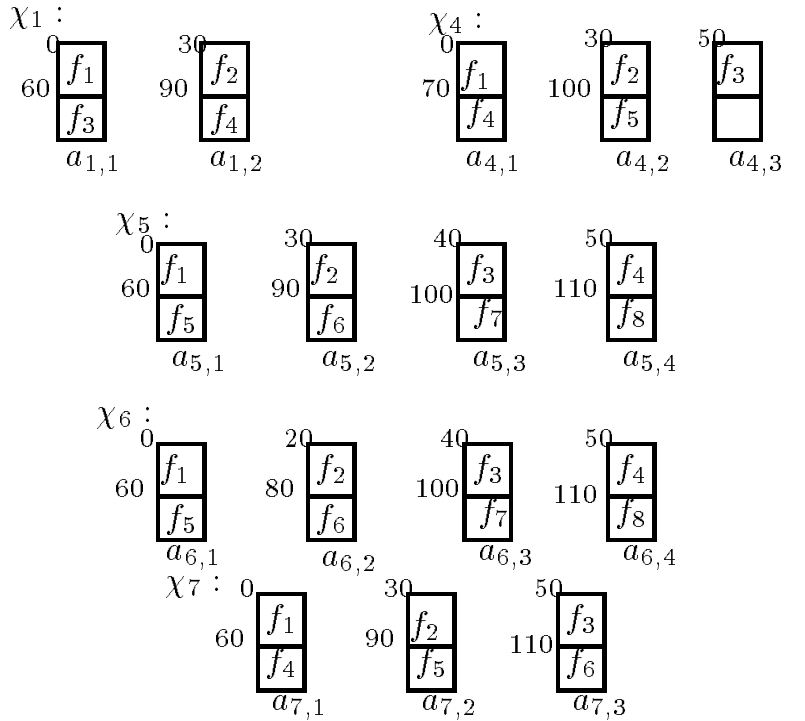

Figure 4: A sample generation of storage sets.

degree of parallelism. Furthermore, conditions (1) and (2) and the acceptibility requirement discussed above must hold. If the application of the above rules yields no storage set, then a storage set whose storage length minimally exceeds the degree of parallelism is selected. In the case of class-two DEs, each DE is made up of one storage set consisting of one AU.

\section{The proposed mapping techniques}

An AU is allocatable to a storage device if the storage device belongs to the device cluster from which the $\mathrm{AU}$ was built. In other words, the bandwidth of the storage device must be equal to the size of the AU's SE. Given an AU, we have a list of storage devices to which it is allocatable. If the AUs and storage devices represent nodes in a graph, then we can construct an edge from an AU to a storage device to which that $\mathrm{AU}$ is allocatable. We must then select one of these storage devices as the most efficient storage for the AU. In order to accomplish this, one must consider the effects of allocating a given AU to all the possible storage devices. If we assign a weight to each of these nodes, then one can, using some criteria, determine the best allocation for a given $\mathrm{AU}$. In order to fairly balance the loads, we need to specify some factors that will help to determine an efficient allocation of an AU. Prominent among these factors are the current status of a storage device with respect to the AUs already allocated, the effect of the free space in the storage device, and the bandwidth of the storage device. The current status function must be defined in terms of an
AU's size and frequency and we call that the expected disk traffic requirement and represent it by a function $\mathcal{F}$. This function must always be defined such that a DE's frequency is emphasized and certain characteristics of the multimedia environment should also be taken into consideration. Through many experiments, we have found that $\mathcal{F}(f, z)=z \frac{1}{1-f}$, where $f, z$ are frequency and size of an AU, respectively, seems to be a good choice. Of course, $\mathcal{F}$ can be defined in many other ways to emphasize special characteristics of an environment. Let $a_{i}$ be an AU, SIZE $\left(a_{i}\right)$ the size of AU $a_{i}$, and FREQ $\left(a_{i}\right)$ the frequency of AU $a_{i}$. The current cumulative traffic requirement of $S_{k}$, assuming that there are a total of $h$ AUs already stored in it, is computed as:

$$
S_{k}^{w}=\sum_{i=1}^{h} \mathcal{F}\left(F \operatorname{RE} Q\left(a_{i}\right), \operatorname{SIZE}\left(a_{i}\right)\right) .
$$

The cumulative traffic requirement of a storage device is an indication of the expected access to the storage device with respect to the AUs allocated to it. Consequently, a reasonable motivation is to allocate the next AU to the storage device with lowest cumulative traffic requirement. However, that factor alone does not determine an efficient storage device to allocate an AU. In order to get a more vivid understanding of the effect of the cumulative traffic requirement, we need to determine the expected disk traffic per unit of allocated space in a storage device. That value indicates the disk traffic exerted per unit of allocated space in a given storage device. We extend the expected disk traffic per unit of allocated space and determine the induced expected disk traffic per unit of allocated space. That is the expected disk traffic per unit of allocated space if the AU under consideration is allocated to a given storage device. We denote as $\mathcal{G}_{k}^{w}$ the induced expected disk traffic per unit of allocated space by an AU on $S_{k}$. After a successful allocation of an AU to $S_{k}, S_{k}^{w}$ becomes $\mathcal{G}_{k}^{w}$. Consequently, for a given $\mathrm{AU}$,

$$
\mathcal{G}_{k, i}^{w}=\frac{S_{k}^{w}+\mathcal{F}\left(F R E Q\left(a_{i}\right), S I Z E\left(a_{i}\right)\right)}{S_{k}^{a}+\operatorname{SIZE}\left(a_{i}\right)} .
$$

It is undoubtably obvious that the amount of free space in a storage device plays a role in determining the current and future utilization of a storage device. The fact that a storage device has a low cumulative traffic requirement relative to another storage device does not convincingly indicate that it is under-utilized relatively. If the storage device with higher cumulative traffic requirement has considerably larger free space, then it is imperative that relative to their available spaces, it is under-utilized. Again, the fact that a storage device has a high cumulative traffic requirement relative to another storage device should not imply an 
automatic rejection of that storage device. If a storage device has a high cumulative traffic requirement but a high bandwidth, then the resultant effect of the cumulative traffic requirement is reduced by the fact that a large chunk of data is retrievable per unit time. Therefore, our mapping goal is to select the storage device that minimizes these factors. It is obvious that, in terms of magnitude, the bandwidth of a storage device is comparatively smaller than its total allocated space and free space (in most cases). Therefore, expressing the impacts of allocated space, free space, and bandwidth with respect to the cumulative traffic requirement requires that the impact from the bandwidth be expressed in such a way that it does not obscure its counterparts. The impact from the bandwidth should be related to the disparity between the bandwidths, i.e., if there is a considerable gap between the smallest and largest bandwidths of the storage devices under consideration, then the bandwidth factor should also reflect that. We represent the sum of these factors as a cost function $\varsigma$. If

$c_{1}=$ cost induced by induced expected disk traffic per unit of allocated space,

$c_{2}=$ cost induced by free space with respect to the cumulative traffic requirement,

$c_{3}=$ bandwidth factor, then,

$$
\varsigma=\left(e_{1} c_{1}+\epsilon_{2} c_{2}\right) \times c_{3}
$$

where $c_{1}=\mathcal{G}_{k}^{w}, c_{2}=\frac{S_{k}^{w}}{S_{k}^{f}}$, and

$c_{3}=1+\frac{\left(e_{3} B W_{\max }-B W_{S_{k}}\right)}{e_{3} B W_{\max }}$.

$B W_{\text {max }}$ is the maximum bandwidth of the storage devices allocatable to an $\mathrm{AU} . B W_{S_{k}}$ is the bandwidth of the storage device currently under consideration from the set of storage devices allocatable to an AU. The coefficients $e_{1}$ and $e_{2}$ are the accentuating values. We use them to emphasize or de-emphasize the relative importance of the corresponding induced cost. We recommend that these coefficients be in the range of zero and 1 . The coefficient $e_{3}$ is used to control the effects of the bandwidth factor and it is determined from the maximum and minimum bandwidths in the system. We recommend that $\epsilon_{3}$ be selected such that the bandwidth factor is in the range 1.5 to 1.9 . In other words, $1.5 \leq 1+\frac{e_{3} B W_{\max }-B W_{S_{k}}}{e_{3} B W_{\max }} \leq 1.9$. For example, given that $\bar{B} W_{\max }=10 \mathrm{MB}$ and $\bar{B} W_{\min }=50 \mathrm{MB}$, if $e_{3}=2$, then the bandwidth factor is in the range of 1.5 to 1.9. On the other hand, if $e_{3}=3$, then the bandwidth factor is in the range of 1.67 to 1.93 . Given an $\mathrm{AU}$ and the $\varsigma$ costs of allocating it to different storage devices, we select the storage device with minimum cost. We denote the fact that an $\mathrm{AU}, a_{i}$, is allocatable to the storage device, $S_{k}$, as $a_{i}-S_{k}$ and the size of each SE in $a_{i}$ as SESIZE $\left(a_{i}\right)$. Therefore, $a_{i}-S_{k}$ if

1. $a_{i} \in \Re^{o n e}$ and

(a) $\operatorname{SESIZE}\left(a_{i}\right)=B W\left(S_{k}\right)$

(b) $S_{k}^{f} \geq S I Z E\left(a_{i}\right)$.

2. $a_{i} \in \Re^{\text {tuo }} \vee a_{i} \in \Re^{\text {three }}$ and

(a) $S_{k}^{f} \geq S I Z E\left(a_{i}\right)$

Our minimization goal is such that given $g$ AUs of a composite multimedia object and $\varsigma_{i, k}$ as the cost of allocating AU $a_{i}$ to the storage device $S_{k}$, therefore,

$$
\begin{gathered}
\operatorname{minimize} \sum_{i=1}^{g} \varsigma_{i, k} \text { where } \exists S_{k}(1 \leq k \leq m) \text { such } \\
\text { that } a_{i}-S_{k} .
\end{gathered}
$$

For example, Table I shows the current values for 6 storage devices, and Table II shows the sizes and frequencies of 5 AUs of a composite multimedia object. After determining allocatabilities of the AUs and computing the $\varsigma$ costs, Table III shows the $\varsigma$ cost of each $\mathrm{AU}$ to the storage device to which it is allocatable. In this simple example, $\epsilon_{1}=e_{2}=1$.

\begin{tabular}{|c|c|c|c|c|c|c|}
\hline & $\mathrm{s}_{1}$ & $\mathrm{~S}_{2}$ & $\mathrm{~S}_{3}$ & $\mathrm{~S}_{4}$ & $\mathrm{~S}_{5}$ & $\mathrm{~S}_{6}$ \\
\hline Free space & $10 \mathrm{MB}$ & $100 \mathrm{MB}$ & $500 \mathrm{MB}$ & $100 \mathrm{MB}$ & $1 \mathrm{MB}$ & $15 \mathrm{MB}$ \\
\hline Allocated space & $5 \mathrm{MB}$ & $120 \mathrm{MB}$ & $10 \mathrm{~KB}$ & $500 \mathrm{~KB}$ & $50 \mathrm{MB}$ & $70 \mathrm{MB}$ \\
\hline $\begin{array}{l}\text { Cur. cum. } \\
\text { traffic reqs. }\end{array}$ & 15728640 & 262144000 & 102400 & 1048576 & 125829120 & 104857600 \\
\hline Bandwidths & $1 \mathrm{MB}$ & $1 \mathrm{MB}$ & $10 \mathrm{MB}$ & $15 \mathrm{MB}$ & $15 \mathrm{MB}$ & $10 \mathrm{MB}$ \\
\hline
\end{tabular}

Table I: A sample of storage devices' current values used for allocation decision.

Table II: A sample of sizes and frequencies of some AUs.

\begin{tabular}{||l|l|l|l|l|l||}
\hline & $a_{1}$ & $a_{2}$ & $a_{3}$ & $a_{4}$ & $a_{5}$ \\
\hline Size & $50 \mathrm{~KB}$ & $75 \mathrm{~KB}$ & $1.5 \mathrm{MB}$ & $100 \mathrm{~KB}$ & $3 \mathrm{MB}$ \\
\hline Frequency & 0.4 & 0.19 & 0.10 & 0.3 & 0.01 \\
\hline
\end{tabular}

Table III: $\varsigma$ costs from Tables I and II.

\begin{tabular}{|l|l|l|l|l|l|l|}
\hline & $S_{1}$ & $S_{2}$ & $S_{3}$ & $S_{4}$ & $S_{5}$ & $S_{6}$ \\
\hline$a_{1}$ & 16.6 & 2810.0 & - & - & - & - \\
\hline$a_{2}$ & 17.5 & 2811.0 & - & - & - & - \\
\hline$a_{3}$ & - & - & 0.2 & - & - & 16.9 \\
\hline$a_{4}$ & - & - & - & 0.1 & 128.1 & - \\
\hline$a_{5}$ & - & - & 0.3 & - & - & 17.2 \\
\hline
\end{tabular}

Consequently, applying the allocation and minimization rules, we have $a_{1}-S_{2}, a_{2}-S_{1}, a_{3}-S_{6}, a_{4}-S_{4}$, $a_{5}-S_{3}$, with a total cost of 2844.8 . 
Figure 5: A sample mapping via Hungarian Method and an unallocatable bipartite graph.

\section{Simulation model and results}

We generated 3 groups of devices where each group has common characteristics such as the bandwidth and size. Each group comprises 10 storage devices. In the results shown below, each composite object consists of a random number of DEs of size between 1 byte and $500 \mathrm{MB}$. The expected retrieval rates range from $.125 \mathrm{~KB} / \mathrm{s}$ to $30 \mathrm{MB} / \mathrm{s}$. Devices in group 1 have size of $100 \mathrm{MB}$ and bandwidth of $1 \mathrm{MB}$, devices in group 2 have size of $75 \mathrm{MB}$ and bandwidth of $750 \mathrm{~KB}$, and devices in group 3 have size of 50MB and bandwidth of $500 \mathrm{~KB}$. Figure 6 shows the distribution, according to frequencies, of the total number of DEs generated. The expected disk traffic function used, given size, $z$, and frequency, $f$, is $\mathcal{F}(f, z)=z\left[\frac{1}{1-f}\right]$.

Figure 7 shows an example final cumulative traffic requirements of the storage devices when a fair mixture of DEs of different frequencies were used. Comparative results were also obtained when more low or high frequency objects were used. Table IV shows the data distribution by percentage in the storage devices after the allocation of the objects. 


\begin{tabular}{|c|c|c|c|c|c|c|c|c|c|c|}
\hline size & $<2 \mathrm{~KB}$ & $<10 \mathrm{~KB}$ & $<100 \mathrm{~KB}$ & $<500 \mathrm{~KB}$ & $<1 \mathrm{MB}$ & $<10 \mathrm{NB}$ & $<50 \mathrm{MB}$ & $<200 \mathrm{MB}$ & $<40 \mathrm{NB}$ & $<500 \mathrm{MB}$ \\
\hline frequency & .160 & .147 & .130 & .120 & .110 & .093 & .080 & .067 & .053 & .040 \\
\hline
\end{tabular}

Figure 6: Distribution of simulation data.

Table IV: A sample percentage data distribution.

\begin{tabular}{|l|l|l|l|l|l|l|l|l|l|l|}
\multicolumn{1}{l|}{} & .040 & .053 & .067 & .080 & .093 & .110 & .120 & .130 & .147 & .160 \\
\hline 1 & 6.3 & 4.6 & 4.6 & 4.1 & 0.0 & 2.4 & 2.6 & 3.7 & 3.5 & 2.9 \\
\hline 2 & 6.9 & 4.2 & 5.7 & 0.0 & 6.6 & 3.5 & 2.2 & 1.6 & 2.6 & 2.4 \\
\hline 3 & 6.8 & 4.8 & 5.6 & 4.1 & 3.4 & 2.8 & 2.5 & 3.2 & 3.2 & 5.9 \\
\hline 4 & 6.1 & 4.9 & 5.5 & 3.6 & 5.8 & 4.3 & 4.4 & 2.1 & 6.9 & 8.4 \\
\hline 5 & 6.0 & 4.0 & 4.1 & 7.5 & 3.6 & 1.2 & 4.7 & 4.9 & 4.1 & 3.5 \\
\hline 6 & 6.8 & 4.8 & 3.0 & 2.2 & 2.3 & 5.4 & 6.5 & 1.3 & 1.1 & 8.3 \\
\hline 7 & 6.8 & 5.2 & 0.0 & 2.5 & 0.0 & 3.4 & 3.9 & 1.2 & 5.3 & 1.0 \\
\hline 8 & 6.6 & 4.7 & 7.1 & 1.9 & 1.5 & 1.5 & 4.9 & 2.4 & 1.5 & 0.9 \\
\hline 9 & 6.6 & 5.5 & 0.0 & 3.8 & 3.6 & 1.3 & 1.3 & 2.2 & 0.9 & 0.9 \\
\hline 10 & 6.4 & 5.1 & 9.3 & 2.5 & 2.2 & 4.5 & 0.0 & 0.5 & 1.5 & 0.0 \\
\hline 11 & 1.4 & 3.7 & 2.3 & 3.5 & 4.2 & 1.6 & 6.7 & 4.3 & 4.8 & 0.8 \\
\hline 12 & 2.5 & 3.8 & 0.0 & 6.1 & 5.0 & 4.1 & 2.3 & 1.1 & 1.7 & 7.5 \\
\hline 13 & 1.4 & 3.0 & 1.4 & 4.3 & 7.6 & 8.4 & 2.5 & 11.1 & 8.1 & 10.2 \\
\hline 14 & 1.4 & 3.7 & 3.1 & 2.3 & 5.8 & 4.0 & 6.0 & 4.2 & 1.6 & 1.6 \\
\hline 15 & 2.0 & 2.4 & 0.0 & 9.8 & 0.0 & 5.8 & 0.0 & 1.7 & 3.4 & 3.3 \\
\hline 16 & 2.8 & 2.8 & 7.9 & 0.0 & 0.0 & 0.0 & 2.5 & 1.9 & 3.0 & 1.5 \\
\hline 17 & 1.4 & 3.3 & 2.9 & 5.0 & 10.2 & 13.3 & 9.2 & 11.2 & 10.2 & 6.7 \\
\hline 18 & 2.1 & 2.4 & 0.0 & 1.0 & 0.0 & 2.5 & 2.6 & 0.3 & 2.2 & 0.0 \\
\hline 19 & 2.3 & 2.8 & 5.5 & 2.3 & 2.2 & 2.8 & 1.5 & 5.9 & 2.7 & 4.4 \\
\hline 20 & 2.3 & 2.2 & 2.3 & 0.7 & 2.9 & 4.6 & 6.0 & 4.7 & 2.2 & 2.2 \\
\hline 21 & 1.8 & 1.4 & 3.0 & 0.0 & 5.3 & 2.6 & 3.2 & 2.5 & 0.0 & 2.7 \\
\hline 22 & 1.5 & 1.6 & 5.2 & 0.0 & 1.5 & 1.2 & 2.9 & 8.7 & 3.3 & 1.4 \\
\hline 23 & 0.7 & 2.0 & 2.6 & 4.1 & 0.0 & 1.6 & 4.7 & 0.0 & 4.0 & 0.9 \\
\hline 24 & 1.3 & 2.5 & 2.1 & 4.4 & 2.4 & 2.2 & 3.5 & 1.2 & 0.0 & 1.9 \\
\hline 25 & 1.1 & 2.2 & 3.2 & 2.9 & 2.0 & 0.0 & 5.2 & 6.9 & 2.8 & 7.5 \\
\hline 26 & 1.6 & 1.0 & 5.4 & 2.9 & 5.8 & 2.4 & 0.8 & 1.5 & 6.3 & 4.4 \\
\hline 27 & 1.8 & 2.0 & 2.8 & 4.5 & 3.4 & 1.4 & 0.0 & 0.7 & 0.7 & 3.2 \\
\hline 28 & 1.6 & 2.7 & 3.3 & 3.4 & 4.1 & 1.4 & 0.9 & 1.1 & 1.0 & 1.5 \\
\hline 29 & 1.9 & 2.5 & 1.0 & 5.5 & 1.7 & 6.8 & 3.1 & 4.5 & 10.4 & 2.6 \\
\hline 30 & 1.8 & 2.0 & 0.9 & 5.1 & 7.1 & 3.1 & 3.4 & 3.2 & 1.1 & 1.2 \\
\hline & & & & & & & & & & \\
\hline
\end{tabular}

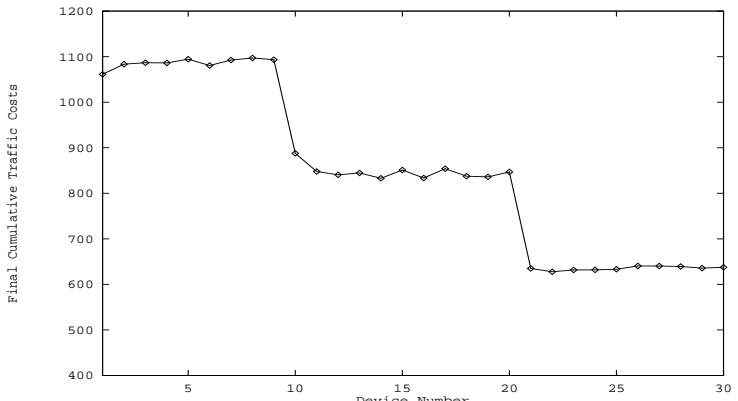

Figure 7: A fair mixture of DEs.

\section{Conclusions}

We have presented a multimedia object model and described the allocation strategy necessary to achieve the real time retrieval requirements of the modeled multimedia objects. We classified the DEs of a composite object into three classes based on their I/O requirements. The allocatability requirements of an $\mathrm{AU}$ to storage devices are defined and the necessary and sufficient criteria for obtaining efficient allocations are described. A cost value based on a disk utilization per allocated space, the amount of free space, and the bandwidth of a storage device are used to determine an efficient allocation and to balance the loads on the storage devices. A bipartite graph model is presented and its characteristics discussed. The bipartite graph forms the basis for multimedia object allocation to storage devices. The Hungarian Method for bipartite matching is used to determine efficient allocation for the AUs of a composite object using the cost values.

\section{References}

[1] C. G. Bell, The mini and macro industries, IEEE Computer, Vol. 17, No. 10, 1984, pp. 1430.

[2] W. Myers, The Competitiveness of U.S.A. Disk Industry, IEEE Computer, Vol. 19, No. 11, 1986, pp. 85-90.

[3] M. Y. Kim, Synchronized Disk Interleaving, IEEE Trans. on Computers, Vol. C-35, Vol. 11, November 1986, pp. 978-988.

[4] M. Livny, S. Khoshafian, H. Boral, MultiDisk Management Algorithms, Proc. 1987 ACM SIGMETRICS Conf. on Measurement and Modeling of Comp. Syst., pp. 69-77.

[5] P. Chen, D. Patterson, Maximizing Performance in a Striped Disk Array, Proc. 1990 ACM SIGARCH 17th Intern. Symp. on Comp. Arch., Seattle, WA, May 1990, pp. 322-331.

[6] J. Zupan, Clustering of Large Data Sets, Technometrics, Vol. 29, Nov., 1987, pp. 497.

[7] A.D. Bell. F.J McErlean. P.M. Stewart, Clustering Related Rules in Databases, The Computer Journal, Vol. 31, June 1988, pp. 253-257.

[8] J.S. Deogun, V.V. Raghava. T.K.W. Tsou, Organization of Clustered Files for Consecutive Retrieval, ACM Trans. on Database Systems, Vol. 9, December 1984, pp. 646-671.

[9] D. P. Anderson, Y. Osawa, A File for Continuous Media, ACM Trans. on Computers, Vol. 10, No. 4, November 1992, pp. 311-337.

[10] P. V. Rangan, H. M. Vin, Designing File Systems for Digital Video and Audio, Proc. 13th ACM Symp. on Operating Systems Principles, Vol. 25, No. 5, Oct., 1991, pp. 81-94.

[11] T.H Cormen, C.E Leiserson, R.L Rivest, Introduction to Algorithms, The MIT Press, 1990.

[12] C.H. Papadimitriou, K. Steiglitz, Combinatorial Optimization, Prentice-Hall, 1982. 